\title{
Equivalent Baseband Channels of Systems Using Envelope Detection
}

\author{
Henning Paul and Karl-Dirk Kammeyer \\ Dedicated to Prof. Dr.-Ing. Werner Rosenkranz on the occasion of his 60th birthday
}

\begin{abstract}
In this paper, the implications of linear channel distortions in systems using envelope detection are reviewed and an equivalent baseband channel is formulated. Finally, the fibre optic channel is treated as a special case to analyse the impairments of Intensity Modulation/Direct Detection (IM/DD) systems employing Single Sideband (SSB) transmission.
\end{abstract}

Keywords tutorial, direct detection, equivalent channel

\section{Introduction}

Direct detection, i.e., square-law detection of the envelope of a received signal, is a technique originally introduced in radio receivers for e.g. broadcast or radar applications, where downconversion is performed inherently by detection of the instantaneous power. In this paper the implications of direct detection in presence of linear channel distortions are reviewed for different analogue modulation schemes such as Double Sideband (DSB), Single Sideband (SSB) and Compatible SSB. Finally, results are transferred to the fibre optic channel, since Orthogonal Frequency Division Multiplexing (OFDM), a digital modulation technique employing a noise-like time domain modulation signal is a promising approach for high-rate long-haul data transmission [1, 2, 3, 4], especially if combined with (Compatible) SSB modulation [5].

The first radio receiver concepts ever developed, the coherer and the detector [6], used envelope detection of the received bandpass signal to perform downconversion. An alternative to detection of instantaneous magnitude is detection of instantaneous power, i.e., the squared magnitude. This is often referred to as square-law detection. Both approaches cause different nonlinear distortion of the signal which have been compared e.g. in [7].

In optical communications, square-law detection is the most commonly used demodulation approach and known as "direct detection" (DD). Considering the electric field of the lightwave - a bandpass signal - as informationconveying quantity, the photo diode at the receiver side is a square-law detector, since it detects instantaneous power, which is proportional to the squared magnitude of the electric field component. Another commonly found approach is to consider the instantaneous power - a lowpass signal - as information-conveying quantity, which requires a different model for the modulator characteristic. This approach has the disadvantage that the transmission channel

Received month 00, 1997.

Dipl.-Ing. Henning Paul, Prof. Dr.-Ing. Karl-Dirk Kammeyer, Department of Communication Engineering, University of Bremen, Otto-Hahn-Allee 1, 28359 Bremen, Germany

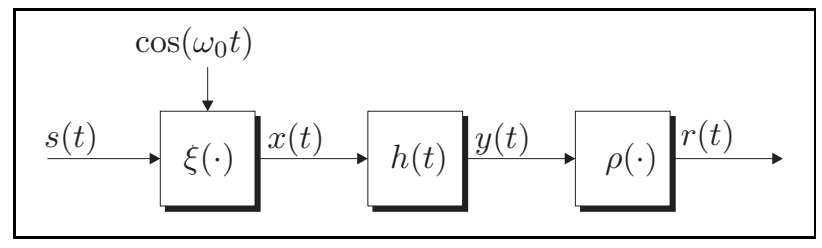

Fig. 1. Block diagram of the system model.

cannot be modelled satisfactorily, since dispersion cannot be described precisely, but only by its effect on the envelope. Due to these restrictions, in the following the former, field-based model will be used.

In Section 2 the system model will be introduced, Section 3 will present the general description of the detection principles. The following Section 4 will apply the results to some examples. In Section 5, the considerations will be generalized to complex-valued modulation, and finally, Section 6 will conclude this paper.

\section{System Model}

Fig. 1 shows the block diagram of the generic system considered in this paper. A real valued modulation signal $s(t)$, consisting of a DC component $\bar{s}$ and the informationbearing, zero-mean signal $\tilde{s}(t)=s(t)-\bar{s}$ is modulated into a bandpass signal $x(t)$ by a nonlinear function $\xi(\cdot)$ employing a carrier $\cos \left(\omega_{0} t\right)$, which is provided by a local oscillator (LO) or in optical applications by a laser. This operation can be described mathematically by

$$
x(t)=\xi\left(s(t), \cos \left(\omega_{0} t\right)\right) .
$$

An ideal modulator should simply move the spectrum $S(j \omega)$ of the time-domain signal $s(t)$ to frequencies $\pm \omega_{0}$, which can be described by the instruction $x(t)=s(t)$. $\cos \left(\omega_{0} t\right)^{1}$. However, in a practical system, the spectrum $X(j \omega)$ will not only be a shifted version of $S(j \omega)$, but also expose other spectral components.

The bandpass signal $x(t)$ passes a linear channel $h(t)$, resulting in a received bandpass signal

$$
y(t)=h(t) * x(t) .
$$

The influence of additive noise will be neglected throughout this paper.

The signal $y(t)$ is then downconverted into baseband by

1 A scaling factor of $\sqrt{2}$ may be introduced to keep the signal powers in baseband and bandpass domain the same, but is omitted for the sake of simplicity. 
envelope detection, either by a magnitude $(\rho(\cdot)=|\cdot|)$ or squared-magnitude $\left(\rho(\cdot)=|\cdot|^{2}\right)$ operation, resulting in a detector output signal

$$
r(t)=\bar{r}+\underbrace{\tilde{h}(t) * \tilde{s}(t)}_{\tilde{r}(t)}+d(t) .
$$

Its DC component is denoted by $\bar{r}$, the informationbearing component by $\tilde{r}(t)$, which is interpreted as a convolution of an equivalent baseband channel $\tilde{h}(t)$ with $\tilde{s}(t)$. $d(t)$ represents an additional interference term (see eq. (6) ), whose properties have an influence on the overall system performance, but are not considered in this paper. In the following, an expression for $\tilde{h}(t)$ will be developed.

\section{Detection principles}

The two incoherent detection principles, magnitude detection and square-law detection, will now be analysed. The latter concept will be dealt with first, since it is easier to handle mathematically.

\subsection{Square-law detection}

The squaring operation on the received bandpass signal $y(t)$ can be expressed using (2) by

$$
i(t)=y^{2}(t)=(h(t) * x(t))^{2} .
$$

Note that bandpass signals always are real valued, thus the squaring operation can be applied directly on $y(t)$ and not its magnitude. If the transmitted baseband signal $x(t)$ is decomposed into carrier and sideband components

$$
x(t)=\bar{x} \cdot \cos \left(\omega_{0} t\right)+\tilde{x}(t),
$$

we find for the squared signal

$$
\begin{aligned}
i(t)= & {\left[h(t) *\left(\bar{x} \cdot \cos \left(\omega_{0} t\right)+\tilde{x}(t)\right)\right]^{2} } \\
= & {\left[\bar{x} \cdot h(t) * \cos \left(\omega_{0} t\right)+h(t) * \tilde{x}(t)\right]^{2} } \\
= & \overbrace{2 \bar{x} \operatorname{Re}\left\{H\left(j \omega_{0}\right) \cdot \mathrm{e}^{j \omega_{0} t}\right\} \cdot(h(t) * \tilde{x}(t))}^{i_{\text {inf }}(t)} \\
& +\bar{x}^{2} \operatorname{Re}\left\{H\left(j \omega_{0}\right) \cdot \mathrm{e}^{j \omega_{0} t}\right\}^{2}+(h(t) * \tilde{x}(t))^{2} .
\end{aligned}
$$

The first term of this sum contains the information-bearing signal component $i_{\text {inf }}(t)$, the second term represents DC and carrier components, while the last one represents distortion due to self-mixing.

As mentioned at the beginning of this Section, if a perfect modulator was used, modulation signal $s(t)$ and bandpass transmit signal were connected by the relation $x(t)=$ $s(t) \cos \left(\omega_{0} t\right)$ and thus, since $\tilde{s}(t)$ is defined to be the zeromean component of $s(t), \tilde{x}(t)=\tilde{s}(t) \cos \left(\omega_{0} t\right)$.

Even though the modulators used in general are not perfect, the above relation can be used if the imperfections of the modulator are moved into a previous stage of the signal chain, that means that a distorted signal $\tilde{\varsigma}(t)$ is used as input of an ideal modulator:

$$
\tilde{x}(t)=\tilde{\varsigma}(t) \cos \left(\omega_{0} t\right) .
$$

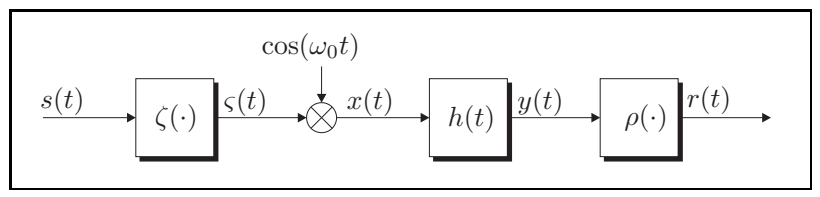

Fig. 2. Modeling the modulator imperfections in the baseband.

Fig. 2 depicts this approach graphically.

Restricting analysis to $i_{\text {inf }}(t)$ in (6) and inserting (7) into it, we get

$$
\begin{aligned}
i_{\text {inf }}(t) & =2 \bar{x} \operatorname{Re}\left\{H_{0} \cdot \mathrm{e}^{j \omega_{0} t}\right\} \cdot(h(t) * \tilde{x}(t)) \\
& =2 \bar{x} \operatorname{Re}\left\{H_{0} \cdot \mathrm{e}^{j \omega_{0} t}\right\} \cdot\left[h(t) *\left(\tilde{\varsigma}(t) \cos \left(\omega_{0} t\right)\right)\right] .
\end{aligned}
$$

Note that $H\left(j \omega_{0}\right)$ has been replaced by $H_{0}$ for simplification of notation.

Using the relation

$$
h(t) *\left(\tilde{\varsigma}(t) \mathrm{e}^{j \omega_{0} t}\right)=\mathrm{e}^{j \omega_{0} t}\left(\left(h(t) \mathrm{e}^{-j \omega_{0} t}\right) * \tilde{\varsigma}(t)\right),
$$

we get

$$
\begin{aligned}
& i_{\text {inf }}(t)=\bar{x} \operatorname{Re}\left\{H_{0} \cdot \mathrm{e}^{j \omega_{0} t}\right\} \\
& \cdot\left[\mathrm{e}^{j \omega_{0} t}\left(\left(h(t) \mathrm{e}^{-j \omega_{0} t}\right) * \tilde{\varsigma}(t)\right)\right. \\
&\left.\quad+\mathrm{e}^{-j \omega_{0} t}\left(\left(h(t) \mathrm{e}^{j \omega_{0} t}\right) * \tilde{\varsigma}(t)\right)\right] .
\end{aligned}
$$

The term $h(t) \mathrm{e}^{-j \omega_{0} t}$ is a frequency shifted version of the physical channel and shall be denoted by $h_{\mathrm{c}}(t)$. Note that when evaluated at frequencies around $0-$ which will be the case in this paper - this frequency shifted channel is identical to the equivalent complex baseband channel of a system using coherent detection [8], which is constructed by concatenation of this frequency shifted channel with a lowpass filter. Due to this, we will not distinguish between the frequency shifted channel and the true equivalent complex baseband channel. Since the physical bandpass channel impulse response $h(t)$ always is real valued, we find that $h_{\mathrm{c}}^{*}(t)=h(t) \mathrm{e}^{j \omega_{0} t}$.

Inserting these definitions into above equation, the expression

$$
\begin{aligned}
i_{\text {inf }}(t)=\bar{x} \operatorname{Re} & \left\{H_{0} \cdot \mathrm{e}^{j \omega_{0} t}\right\} \cdot\left[\mathrm{e}^{j \omega_{0} t}\left(h_{\mathrm{c}}(t) * \tilde{\varsigma}(t)\right)\right. \\
& \left.+\mathrm{e}^{-j \omega_{0} t}\left(h_{\mathrm{c}}^{*}(t) * \tilde{\varsigma}(t)\right)\right]
\end{aligned}
$$

is gained. This equation can be rearranged into the alternate form

$$
\begin{aligned}
i_{\text {inf }}(t)=\bar{x} & \operatorname{Re}\left\{H_{0} \cdot \mathrm{e}^{j \omega_{0} t}\right\} \\
\cdot[ & \cos \left(\omega_{0} t\right)\left(\operatorname{Re}\left\{h_{\mathrm{c}}(t)\right\} * \tilde{\varsigma}(t)\right) \\
& \left.\quad-\sin \left(\omega_{0} t\right)\left(\operatorname{Im}\left\{h_{\mathrm{c}}(t)\right\} * \tilde{\varsigma}(t)\right)\right],
\end{aligned}
$$

which has the advantage of consisting only of real valued convolutions. A modified block diagram of the overall system based on this representation is depicted in Fig. 3 .

Replacing the real part operation in (11) by

$$
\operatorname{Re}\left\{H_{0} \cdot \mathrm{e}^{j \omega_{0} t}\right\}=\frac{1}{2}\left(H_{0} \cdot \mathrm{e}^{j \omega_{0} t}+H_{0}^{*} \cdot \mathrm{e}^{-j \omega_{0} t}\right)
$$




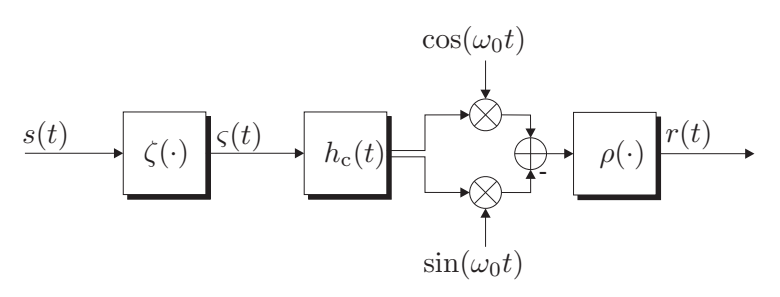

Fig. 3. Block diagram of the modified system representation.

where $(\cdot)^{*}$ denotes the complex conjugate, the exponential terms can be collected, resulting in

$$
\begin{aligned}
i_{\text {inf }}(t)= & \bar{x} \frac{1}{2}\left(H_{0} \cdot \mathrm{e}^{j \omega_{0} t}+H_{0}^{*} \cdot \mathrm{e}^{-j \omega_{0} t}\right) \\
\cdot\left[\mathrm{e}^{j \omega_{0} t}\left(h_{\mathrm{c}}(t) * \tilde{\varsigma}(t)\right)\right. & \left.\quad+\mathrm{e}^{-j \omega_{0} t}\left(h_{\mathrm{c}}^{*}(t) * \tilde{\varsigma}(t)\right)\right] \\
=\frac{\bar{x}}{2} & {\left[H_{0} \cdot \mathrm{e}^{j 2 \omega_{0} t}\left(h_{\mathrm{c}}(t) * \tilde{\varsigma}(t)\right)\right.} \\
& +H_{0} \cdot\left(h_{\mathrm{c}}^{*}(t) * \tilde{\varsigma}(t)\right) \\
& +H_{0}^{*} \cdot\left(h_{\mathrm{c}}(t) * \tilde{\varsigma}(t)\right) \\
& \left.+H_{0}^{*} \cdot \mathrm{e}^{-j 2 \omega_{0} t}\left(h_{\mathrm{c}}^{*}(t) * \tilde{\varsigma}(t)\right)\right] .
\end{aligned}
$$

This signal obviously contains spectral components at twice the carrier frequency $2 \omega_{0}$. These components either cannot propagate as in optical communications or are eliminated by a lowpass filter of appropriate cut-off frequency, which would be included in the function $\rho(y(t))$. Its output can be described by

$$
\begin{aligned}
\tilde{r}(t)= & \frac{\bar{x}}{2}\left[H_{0} \cdot\left(h_{\mathrm{c}}^{*}(t) * \tilde{\varsigma}(t)\right)\right. \\
& \left.\quad+H_{0}^{*} \cdot\left(h_{\mathrm{c}}(t) * \tilde{\varsigma}(t)\right)\right] \\
= & \frac{\bar{x}}{2}\left[H_{0} h_{\mathrm{c}}^{*}(t)+H_{0}^{*} h_{\mathrm{c}}(t)\right] * \tilde{\varsigma}(t),
\end{aligned}
$$

which can also be represented as

$$
\tilde{r}(t)=\bar{x} \operatorname{Re}\left\{H_{0}^{*} h_{\mathrm{c}}(t)\right\} * \tilde{\varsigma}(t) .
$$

\subsection{Magnitude detection}

To find a solution for the case of magnitude detection, the approach above has to be modified slightly. Since the magnitude $|y(t)|$ can be expressed by

$$
|y(t)|=\sqrt{i(t)}
$$

above expression is now expanded into a Taylor series. For this reason, the DC component of $i(t)$ is separated. Using

$$
\begin{aligned}
& \bar{x}^{2} \operatorname{Re}\left\{H_{0} \cdot \mathrm{e}^{j \omega_{0} t}\right\}^{2} \\
= & \frac{\bar{x}^{2}}{4}\left|H_{0} \cdot \mathrm{e}^{j \omega_{0} t}+H_{0}^{*} \cdot \mathrm{e}^{-j \omega_{0} t}\right|^{2} \\
= & \frac{\bar{x}^{2}}{2}\left(\left|H_{0}\right|^{2}+\operatorname{Re}\left\{H_{0}^{2} \cdot \mathrm{e}^{j 2 \omega_{0} t}\right\}\right),
\end{aligned}
$$

(6) can be rearranged into

$$
\begin{aligned}
i(t) & =\frac{\bar{x}^{2}}{2}\left|H_{0}\right|^{2}+\frac{\bar{x}^{2}}{2} \operatorname{Re}\left\{H_{0}^{2} \cdot \mathrm{e}^{j 2 \omega_{0} t}\right\} \\
& +2 \bar{x} \operatorname{Re}\left\{H_{0} \cdot \mathrm{e}^{j \omega_{0} t}\right\} \cdot(h(t) * \tilde{x}(t)) \\
& +(h(t) * \tilde{x}(t))^{2} .
\end{aligned}
$$

An expression $\sqrt{a+b(t)}$ with $b(t)$ being zero mean can be expanded into a Taylor series which, terminated after the quadratic term, reads

$$
\begin{aligned}
\sqrt{a+b(t)} & \approx \sqrt{a}\left[1+\frac{1}{2} \frac{b(t)}{a}-\frac{1}{8} \frac{b^{2}(t)}{a^{2}}\right] \\
& =\sqrt{a}+\frac{1}{2 \sqrt{a}} b(t)-\frac{b^{2}(t)}{8 a^{\frac{3}{2}}} .
\end{aligned}
$$

Second and third term of the sum in (20) have zero mean, while the last one, $(h(t) * \tilde{x}(t))^{2}$ has not. Its mean represents the received sideband power and can be calculated in frequency domain:

$$
\begin{aligned}
P_{\mathrm{sb}} & =\left.\mathcal{F}\left\{(h(t) * \tilde{x}(t))^{2}\right\}\right|_{\omega=0} \\
& =\frac{1}{2 \pi} \int_{-\infty}^{\infty}|H(j \omega) \tilde{X}(j \omega)|^{2} d \omega .
\end{aligned}
$$

Applying (21) to (18) and defining the received carrier power $P_{\mathrm{c}}=\frac{\bar{x}^{2}}{2}\left|H_{0}\right|^{2}$, we get

$$
\begin{aligned}
& |y(t)|=\sqrt{i(t)} \\
& \approx \sqrt{P_{\mathrm{c}}+P_{\mathrm{sb}}} \\
& +\frac{1}{2 \sqrt{P_{\mathrm{c}}+P_{\mathrm{sb}}}}\left[\frac{\bar{x}^{2}}{2} \operatorname{Re}\left\{H_{0}^{2} \cdot \mathrm{e}^{j 2 \omega_{0} t}\right\}\right. \\
& +2 \bar{x} \operatorname{Re}\left\{H_{0} \cdot \mathrm{e}^{j \omega_{0} t}\right\} \cdot(h(t) * \tilde{x}(t)) \\
& \left.+(h(t) * \tilde{x}(t))^{2}-P_{\mathrm{sb}}\right] \\
& -\frac{1}{8{\sqrt{P_{\mathrm{c}}+P_{\mathrm{sb}}}}^{3}}\left[\frac{\bar{x}^{2}}{2} \operatorname{Re}\left\{H_{0}^{2} \cdot \mathrm{e}^{j 2 \omega_{0} t}\right\}\right. \\
& +2 \bar{x} \operatorname{Re}\left\{H_{0} \cdot \mathrm{e}^{j \omega_{0} t}\right\} \cdot(h(t) * \tilde{x}(t)) \\
& \left.+(h(t) * \tilde{x}(t))^{2}-P_{\mathrm{sb}}\right]^{2} .
\end{aligned}
$$

Expanding the square operation and restricting analysis to the information-bearing signal component $y_{\text {inf }}(t)$, i.e. the sum of all terms containing $h(t) * \tilde{x}(t)$ to the power of 1 , we get

$$
\begin{aligned}
& y_{\text {inf }}(t)=\frac{\bar{x}}{\sqrt{P_{\mathrm{c}}+P_{\mathrm{sb}}}}\left[\operatorname{Re}\left\{H_{0} \cdot \mathrm{e}^{j \omega_{0} t}\right\}-\frac{1}{8\left(P_{\mathrm{c}}+P_{\mathrm{sb}}\right)}\right. \\
& \cdot\left(2 \bar{x}^{2} \operatorname{Re}\left\{H_{0}^{2} \cdot \mathrm{e}^{j 2 \omega_{0} t}\right\} \operatorname{Re}\left\{H_{0} \cdot \mathrm{e}^{j \omega_{0} t}\right\}\right. \\
&\left.\left.-4 P_{\mathrm{sb}} \operatorname{Re}\left\{H_{0} \cdot \mathrm{e}^{j \omega_{0} t}\right\}\right)\right] \cdot(h(t) * \tilde{x}(t)) \cdot(24)
\end{aligned}
$$

With

$$
\operatorname{Re}\left\{H_{0}^{2} \cdot \mathrm{e}^{j 2 \omega_{0} t}\right\} \operatorname{Re}\left\{H_{0} \cdot \mathrm{e}^{j \omega_{0} t}\right\}
$$




$$
\begin{aligned}
= & \frac{1}{2} \operatorname{Re}\left\{H_{0}^{3} \cdot \mathrm{e}^{j 3 \omega_{0} t}\right\} \\
& +\frac{1}{2}\left|H_{0}\right|^{2} \operatorname{Re}\left\{H_{0} \cdot \mathrm{e}^{j \omega_{0} t}\right\},
\end{aligned}
$$

(24) can be rearranged into

$$
\begin{aligned}
& y_{\text {inf }}(t)= \frac{\bar{x}}{\sqrt{P_{\mathrm{c}}+P_{\mathrm{sb}}}}\left[\operatorname{Re}\left\{H_{0} \cdot \mathrm{e}^{j \omega_{0} t}\right\}\right. \\
&-\frac{1}{8\left(P_{\mathrm{c}}+P_{\mathrm{sb}}\right)}\left(\bar{x}^{2} \operatorname{Re}\left\{H_{0}^{3} \cdot \mathrm{e}^{j 3 \omega_{0} t}\right\}\right. \\
&\left.\left.+\left(\bar{x}^{2}\left|H_{0}\right|^{2}-4 P_{\mathrm{sb}}\right) \operatorname{Re}\left\{H_{0} \cdot \mathrm{e}^{j \omega_{0} t}\right\}\right)\right] \\
& \cdot(h(t) * \tilde{x}(t)) .
\end{aligned}
$$

The term $\operatorname{Re}\left\{H_{0}^{3} \cdot \mathrm{e}^{j 3 \omega_{0} t}\right\}$ causes a shift of the bandpass spectrum located around $\omega_{0}$ to frequencies $2 \omega_{0}$ and $4 \omega_{0}$ which is suppressed and therefore can be ignored, resulting in an expression

$$
\begin{array}{r}
y_{\mathrm{inf}}(t)=\frac{\bar{x}}{\sqrt{P_{\mathrm{c}}+P_{\mathrm{sb}}}}\left[1-\frac{\bar{x}^{2}\left|H_{0}\right|^{2}-4 P_{\mathrm{sb}}}{8\left(P_{\mathrm{c}}+P_{\mathrm{sb}}\right)}\right] \\
\cdot \operatorname{Re}\left\{H_{0} \cdot \mathrm{e}^{j \omega_{0} t}\right\} \cdot(h(t) * \tilde{x}(t)) .
\end{array}
$$

By insertion of the definitions $P_{\mathrm{c}}=\frac{1}{2} \bar{x}^{2}\left|H_{0}\right|^{2}$ and $H_{0}=$ $\left|H_{0}\right| \mathrm{e}^{j \varphi_{0}}$, above expression can be rearranged into

$$
\begin{array}{r}
y_{\text {inf }}(t)=\frac{\sqrt{2}}{\sqrt{1+P_{\mathrm{sb}} / P_{\mathrm{c}}}}\left[1-\frac{1-2 P_{\mathrm{sb}} / P_{\mathrm{c}}}{4\left(1+P_{\mathrm{sb}} / P_{\mathrm{c}}\right)}\right] \\
\cdot \operatorname{Re}\left\{\mathrm{e}^{j \varphi_{0}} \cdot \mathrm{e}^{j \omega_{0} t}\right\} \cdot(h(t) * \tilde{x}(t)) .
\end{array}
$$

Using the same relations as applied in (10), the filtered information-bearing output signal $\tilde{r}(t)$ after suppression of spectral components at twice the carrier frequency can be described by

$$
\begin{gathered}
\tilde{r}(t)=\frac{\sqrt{2}}{\sqrt{1+P_{\mathrm{sb}} / P_{\mathrm{c}}}}\left[1-\frac{1-2 P_{\mathrm{sb}} / P_{\mathrm{c}}}{4\left(1+P_{\mathrm{sb}} / P_{\mathrm{c}}\right)}\right] \\
\cdot \operatorname{Re}\left\{\mathrm{e}^{-j \varphi_{0}} h_{\mathrm{c}}(t)\right\} * \tilde{\varsigma}(t)
\end{gathered}
$$

This result is similar to (17), but differs in several aspects: It does not directly depend on $\bar{x}$ and $\left|H_{0}\right|$, but on the ratio $P_{\mathrm{sb}} / P_{\mathrm{c}}$ of sideband vs. carrier power. Furthermore the real part operation is not performed on $H_{0}^{*} h_{\mathrm{c}}(t)$, but on $\mathrm{e}^{-j \varphi_{0}} h_{\mathrm{c}}(t)$, which means that the channel gain does not contribute in a square fashion to the equivalent channel, but only linearly.

For realistic power ratios $0.1 \leq P_{\mathrm{sb}} / P_{\mathrm{c}} \leq 10$, we find that

$$
\frac{\sqrt{2}}{\sqrt{1+P_{\mathrm{sb}} / P_{\mathrm{c}}}}\left[1-\frac{1-2 P_{\mathrm{sb}} / P_{\mathrm{c}}}{4\left(1+P_{\mathrm{sb}} / P_{\mathrm{c}}\right)}\right] \approx 1 .
$$

If the order of the Taylor series expansion in (18) is increased, this equality is approximated even closer, so that the filtered detector output can be given by

$$
\tilde{r}(t)=\operatorname{Re}\left\{\mathrm{e}^{-j \varphi_{0}} h_{\mathrm{c}}(t)\right\} * \tilde{\varsigma}(t) .
$$

This shows that both detection principles result in equivalent baseband channels which differ only in a factor $\bar{x}\left|H_{0}\right|$, therefore it is sufficient to restrict further analysis to one of them. For simplicity reasons, the case of square-law detection is chosen. Please note that the power of the interference terms which have been neglected in above derivations might differ significantly for both detection principles.

\section{Examples of equivalent channels}

If an ideal modulator is assumed, i.e. $\varsigma(t)=s(t)$, in case of square-law detection the equivalent baseband channel $\tilde{h}(t)$ acting on the zero-mean signal $\tilde{s}(t)$ reads

$$
\tilde{h}(t)=\bar{x} \operatorname{Re}\left\{H_{0}^{*} h_{\mathrm{c}}(t)\right\},
$$

or, in frequency domain

$$
\tilde{H}(j \omega)=\frac{\bar{x}}{2}\left(H_{0}^{*} H_{\mathrm{c}}(j \omega)+H_{0} H_{\mathrm{c}}^{*}(-j \omega)\right) .
$$

\subsection{2-path fading channel}

For simplicity reasons, the 2-path fading channel

$$
h(t)=a_{1} \delta_{0}\left(t-\tau_{1}\right)+a_{2} \delta_{0}\left(t-\tau_{2}\right),
$$

well-known from wireless communication, is considered as a first example. Due to the sifting property of the delta function, its corresponding complex baseband channel is described by

$$
\begin{aligned}
h_{\mathrm{c}}(t) & =h(t) \mathrm{e}^{j \omega_{0} t} \\
& =a_{1} \mathrm{e}^{j \omega_{0} \tau_{1}} \delta_{0}\left(t-\tau_{1}\right)+a_{2} \mathrm{e}^{j \omega_{0} \tau_{2}} \delta_{0}\left(t-\tau_{2}\right) \\
& =c_{1} \delta_{0}\left(t-\tau_{1}\right)+c_{2} \delta_{0}\left(t-\tau_{2}\right) .
\end{aligned}
$$

According to (32), the equivalent baseband channel in this case reads

$$
\begin{aligned}
\tilde{h}(t)= & \bar{x} \operatorname{Re}\left\{\left(c_{1}^{*}+c_{2}^{*}\right)\left(c_{1} \delta_{0}\left(t-\tau_{1}\right)+c_{2} \delta_{0}\left(t-\tau_{2}\right)\right)\right\} \\
= & \bar{x}\left(\left|c_{1}\right|^{2}+\operatorname{Re}\left\{c_{1} c_{2}^{*}\right\}\right) \delta_{0}\left(t-\tau_{1}\right) \\
& +\bar{x}\left(\left|c_{2}\right|^{2}+\operatorname{Re}\left\{c_{1} c_{2}^{*}\right\}\right) \delta_{0}\left(t-\tau_{2}\right)
\end{aligned}
$$

therefore also is a 2-path fading channel, but with modified, real valued channel coefficients.

\subsection{The fibre optical channel}

All the considerations made above can also be applied to fibre optical transmission systems. In this case, the carrier frequency $\omega_{0}=2 \pi f_{0}$ lies at approximately $2 \pi \cdot 200 \mathrm{THz}$ and suppression of spectral components at multiples of the carrier frequency in the electrical domain takes place inherently, but the analysis above is still valid in this case.

The group velocity dispersion (GVD) governed, lossless linear fibre optical channel [9] can be expressed in frequency domain by an allpass channel with bandpass domain representation

$$
H(j \omega)=\mathrm{e}^{j b(\omega)},
$$


where $b(\omega)$ is a polynomial in $\omega$. Around $\omega_{0}$, this polynomial can be truncated after the quadratic term in good approximation, resulting in

$$
H(j \omega)=\mathrm{e}^{j\left(b_{2} \omega^{2}+b_{1} \omega+b_{0}\right)} .
$$

Its complex baseband channel can be constructed by

$$
\begin{aligned}
H_{\mathrm{c}}(j \omega) & =H\left(j\left(\omega+\omega_{0}\right)\right) \\
& =\mathrm{e}^{j\left(b_{2}\left(\omega+\omega_{0}\right)^{2}+b_{1}\left(\omega+\omega_{0}\right)+b_{0}\right)} \\
& =H_{0} \cdot \mathrm{e}^{-j \tau \omega} \mathrm{e}^{j b_{2} \omega^{2}}
\end{aligned}
$$

with $H_{0}=\mathrm{e}^{j\left(b_{2} \omega_{0}^{2}+b_{1} \omega_{0}+b_{0}\right)}$ and $\tau=-2 b_{2} \omega_{0}-b_{1}$. Inserting this into (33), we get

$$
\begin{aligned}
\tilde{H}(j \omega)= & \frac{\bar{x}}{2}\left(H_{0}^{*} H_{0} \cdot \mathrm{e}^{-j \tau \omega} \mathrm{e}^{j b_{2} \omega^{2}}\right. \\
& \left.+H_{0} H_{0}^{*} \cdot \mathrm{e}^{-j \tau \omega} \mathrm{e}^{-j b_{2} \omega^{2}}\right) \\
= & \bar{x} \mathrm{e}^{-j \tau \omega} \cos \left(b_{2} \omega^{2}\right) .
\end{aligned}
$$

This channel has linear phase, but has a frequency selective amplitude response and exposes zeros at $\omega=$ $\pm \sqrt{n \pi / b_{2}+\pi /\left(2 b_{2}\right)}$, which is known as "power fading". This effect has been derived in [10] and limits the performance of optical transmission systems significantly.

\subsection{The fibre optical channel with sideband filtering}

The "power fading" effect in the previous example causes severe distortions of the transmitted signal. For this reason, Single Sideband (SSB) transmission has been proposed [11]. At the transmitter, one sideband of the bandpass signal is suppressed by means of an optical filter. The frequency response of the complex baseband channel resulting from the concatenation of optical filter and fibre can be expressed by

$$
H_{\mathrm{c}}(j \omega)=\left\{\begin{array}{cl}
H_{0} \cdot \mathrm{e}^{-j \tau \omega} \mathrm{e}^{j b_{2} \omega^{2}}, & \omega \geq 0 \\
0, & \text { else }
\end{array}\right.
$$

if the lower sideband is assumed to be suppressed perfectly. In this case, the equivalent baseband channel is easily found to be

$$
\tilde{H}(j \omega)=\frac{\bar{x}}{2} \mathrm{e}^{-j \tau \omega}\left\{\begin{array}{cc}
\mathrm{e}^{-j b_{2} \omega^{2}}, & \omega<0 \\
1, & \omega=0 \\
\mathrm{e}^{j b_{2} \omega^{2}}, & \omega>0
\end{array},\right.
$$

thus has allpass characteristic and maintains the phase response of the physical channel for positive frequencies, no nulling occurs.

\section{Complex valued modulation}

In the previous considerations, we were restricting our analysis to real valued (virtual) modulation signals $\varsigma(t)$. However, it is possible to design a modulator $\xi(\cdot)$ that modulates a real valued signal $s(t)$ into a bandpass signal

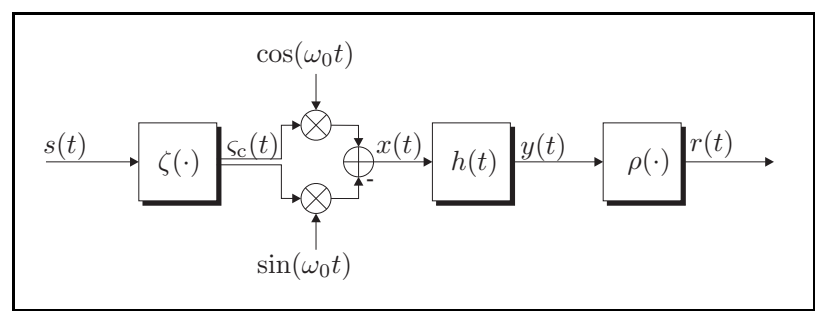

Fig. 4. Complex valued extension of the modulator model.

$x(t)$ that cannot be described by a real valued baseband signal $\varsigma(t)$, but requires complex-valued representation

$$
x(t)=\operatorname{Re}\left\{\varsigma_{\mathrm{c}}(t)\right\} \cos \left(\omega_{0} t\right)-\operatorname{Im}\left\{\varsigma_{\mathrm{c}}(t)\right\} \sin \left(\omega_{0} t\right)
$$

with $\varsigma_{\mathrm{c}}(t)$ being complex-valued. Fig. 4 depicts this complex-valued extension of the modulator model.

Prominent examples for modulators requiring this model are phase modulator and frequency modulator, but also the single sideband (SSB) modulator. In a single sideband signal, real part $\varsigma^{\prime}(t)=\operatorname{Re}\left\{\varsigma_{\mathrm{c}}(t)\right\}$ and imaginary part $\varsigma^{\prime \prime}(t)=\operatorname{Im}\left\{\varsigma_{c}(t)\right\}$ are connected [8] via the relation

$$
\varsigma^{\prime \prime}(t)=\mathcal{H}\left\{\varsigma^{\prime}(t)\right\},
$$

where $\mathcal{H}\{\cdot\}$ denotes the Hilbert transform. If $\varsigma^{\prime}(t)=s(t)$, the resulting overall system is equivalent to sideband suppression in the bandpass domain as e.g. in Section 4.3, as will be shown later.

\subsection{Compatible single sideband}

There have been endeavours in the 1960s [12, 13, 14] to craft a complex-valued single sideband signal $\varsigma_{\mathrm{c}}(t)$ such that its magnitude $\left|\varsigma_{\mathrm{c}}(t)\right|$ represents the modulation signal $s(t)$ perfectly, i.e., that an envelope detector is able to reconstruct it perfectly - as long as the channel $H(j \omega)$ exposes no frequency selectivity.

This modulation has been entitled "Compatible SSB" and has recently been proposed for fibre optical transmission [5]. It can be shown that phase modulation of $s(t)$ with $\mathcal{H}\{\log s(t)\}$ resulting in

$$
\varsigma_{c}(t)=s(t) \cdot \mathrm{e}^{j \mathcal{H}\{\log s(t)\}}
$$

fulfills this criterion if it can be ensured that $\log s(t)$ exists, i.e., $s(t)>0$. Note that this multiplication is not a linear operation, in contrast to addition of $j$ times the Hilbert transform, which can be represented by linear filtering with an impulse response $g_{\mathcal{H}}(t)$. In fact, it can be interpreted as a phase modulation of $s(t)$, which causes the spectrum to be spread to infinity in positive direction, whereas the lower sideband is cancelled.

Real and imaginary part of this complex baseband signal are given by

$$
\begin{aligned}
\varsigma^{\prime}(t) & =s(t) \cdot \cos (\mathcal{H}\{\log s(t)\}) \\
\varsigma^{\prime \prime}(t) & =s(t) \cdot \sin (\mathcal{H}\{\log s(t)\})
\end{aligned}
$$

and thus

$$
x(t)=s(t) \cdot\left[\cos (\mathcal{H}\{\log s(t)\}) \cos \left(\omega_{0} t\right)\right.
$$




$$
\left.-\sin (\mathcal{H}\{\log s(t)\}) \sin \left(\omega_{0} t\right)\right]
$$

\subsection{Downconversion in the complex case}

In order to extend our model (8) for complex-valued $\tilde{\varsigma}_{\mathrm{c}}(t)$, we have to reformulate (5), since now the transmitted carrier can have arbitrary phase:

$$
x(t)=\operatorname{Re}\left\{\bar{x}_{\mathrm{c}} \cdot \mathrm{e}^{j \omega_{0} t}\right\}+\tilde{x}(t) .
$$

Using this equation, we get for the information-bearing component of the detector output

$$
\begin{aligned}
i_{\text {inf }}(t)= & 2 \operatorname{Re}\left\{\bar{x}_{\mathrm{c}} H_{0} \cdot \mathrm{e}^{j \omega_{0} t}\right\} \\
& \cdot\left[h(t) *\left(\tilde{\varsigma}^{\prime}(t) \cos \left(\omega_{0} t\right)-\tilde{\varsigma}^{\prime \prime}(t) \sin \left(\omega_{0} t\right)\right)\right] \\
= & \left(\bar{x}_{\mathrm{c}} H_{0} \cdot \mathrm{e}^{j \omega_{0} t}+\bar{x}_{\mathrm{c}}^{*} H_{0}^{*} \cdot \mathrm{e}^{-j \omega_{0} t}\right) \\
& \cdot \frac{1}{2}\left(\mathrm{e}^{j \omega_{0} t} h_{\mathrm{c}}(t) * \tilde{\varsigma}_{\mathrm{c}}(t)+\mathrm{e}^{-j \omega_{0} t} h_{\mathrm{c}}^{*}(t) * \tilde{\varsigma}_{\mathrm{c}}^{*}(t)\right) .
\end{aligned}
$$

After suppression of components at twice the carrier frequency, we get

$$
\begin{aligned}
\tilde{r}(t) & =\frac{1}{2}\left(\bar{x}_{\mathrm{c}}^{*} H_{0}^{*} h_{\mathrm{c}}(t) * \tilde{\varsigma}_{\mathrm{c}}(t)+\bar{x}_{\mathrm{c}} H_{0} h_{\mathrm{c}}^{*}(t) * \tilde{\varsigma}_{\mathrm{c}}^{*}(t)\right) \\
& =\operatorname{Re}\left\{\bar{x}_{\mathrm{c}}^{*} H_{0}^{*} h_{\mathrm{c}}(t) * \tilde{\varsigma}_{\mathrm{c}}(t)\right\} .
\end{aligned}
$$

Note that here an equivalent baseband channel $\tilde{h}(t)$ cannot be formulated, since the real part operator $\operatorname{Re}\{\cdot\}$ is not linear. However, it is possible to describe the channel using a "widely linear" approach. In widely linear system descriptions, real and imaginary part of the system input are treated as independent inputs, the same holds for real and imaginary part of the system output. If this approach is applied to $\tilde{\varsigma}_{\mathrm{c}}(t)$, even though in this case real and imaginary part are not independent, as implied by (44), it is possible to formulate an equivalent baseband channel for each of it, according to

$$
\tilde{r}(t)=\tilde{h}^{\prime}(t) * \tilde{\varsigma}^{\prime}(t)+\tilde{h}^{\prime \prime}(t) * \tilde{\varsigma}^{\prime \prime}(t) .
$$

For the real part $\tilde{\varsigma}^{\prime}(t)$, the channel $\tilde{h}^{\prime}(t)$ is identical to the real valued case above

$$
\tilde{h}^{\prime}(t)=\operatorname{Re}\left\{\bar{x}_{\mathrm{c}}^{*} H_{0}^{*} h_{\mathrm{c}}(t)\right\},
$$

while $\tilde{h}^{\prime \prime}(t)$ can be found easily by replacing $\tilde{\varsigma}_{\mathrm{c}}(t)$ by $j \tilde{\varsigma}^{\prime \prime}(t)$ in $(51)$

$$
\begin{aligned}
\tilde{h}^{\prime \prime}(t) & =\operatorname{Re}\left\{j \bar{x}_{\mathrm{c}}^{*} H_{0}^{*} h_{\mathrm{c}}(t)\right\} \\
& =-\operatorname{Im}\left\{\bar{x}_{\mathrm{c}}^{*} H_{0}^{*} h_{\mathrm{c}}(t)\right\} .
\end{aligned}
$$

If now the dependence (44) is introduced, we get for the output

$$
\begin{aligned}
\tilde{r}(t) & =\tilde{h}^{\prime}(t) * \tilde{\varsigma}^{\prime}(t)+\tilde{h}^{\prime \prime}(t) * \mathcal{H}\left\{\tilde{\varsigma}^{\prime}(t)\right\} \\
& =\left[\tilde{h}^{\prime}(t)+g_{\mathcal{H}}(t) * \tilde{h}^{\prime \prime}(t)\right] * \tilde{\varsigma}^{\prime}(t),
\end{aligned}
$$

where $g_{\mathcal{H}}(t)$ is the impulse response of the Hilbert transformer. Writing down the bracketed expression in frequency domain, we get

$$
\tilde{H}_{\mathrm{SSB}}(j \omega)=\mathcal{F}\left\{\tilde{h}^{\prime}(t)+g_{\mathcal{H}}(t) * \tilde{h}^{\prime \prime}(t)\right\}
$$

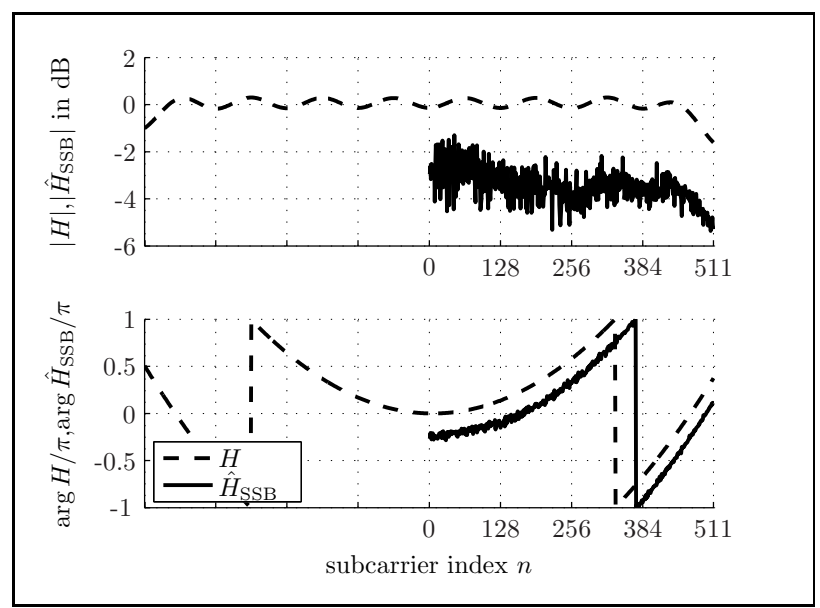

Fig. 5. Channel estimation in an optical OFDM system using electrical SSB modulation.

$$
\begin{aligned}
= & \frac{1}{2}\left[\bar{x}_{\mathrm{c}}^{*} H_{0}^{*} H_{\mathrm{c}}(j \omega)+j G_{\mathcal{H}}(j \omega) \bar{x}_{\mathrm{c}}^{*} H_{0}^{*} H_{\mathrm{c}}(j \omega)\right. \\
& \left.+\bar{x}_{\mathrm{c}} H_{0} H_{\mathrm{c}}^{*}(-j \omega)-j G_{\mathcal{H}}(j \omega) \bar{x}_{\mathrm{c}} H_{0} H_{\mathrm{c}}^{*}(-j \omega)\right],
\end{aligned}
$$

using the frequency domain definition of the Hilbert transformer $G_{\mathcal{H}}(j \omega)=-j \operatorname{sgn}(\omega)$ with $\operatorname{sgn}(\cdot)$ being the sign function, this expression can be simplified to

$$
\begin{aligned}
\tilde{H}_{\mathrm{SSB}}(j \omega) & =\mathcal{F}\left\{\tilde{h}^{\prime}(t)+g_{\mathcal{H}}(t) * \tilde{h}^{\prime \prime}(t)\right\} \\
& =\left\{\begin{array}{cc}
\bar{x}_{\mathrm{c}}^{*} H_{0}^{*} H_{\mathrm{c}}(j \omega), & \omega>0 \\
0, & \omega=0 \\
\bar{x}_{\mathrm{c}} H_{0} H_{\mathrm{c}}^{*}(-j \omega), & \omega<0
\end{array} .\right.
\end{aligned}
$$

This result resembles the result (42) obtained for the filtered fibre optical channel, but is more general and applies to arbitrary channels $H_{\mathrm{c}}(j \omega)$. Nevertheless, for the special case of the fibre optical channel, its effect on the signal is the same, nulling of the channel frequency response is avoided. This emphasizes the fact that complex-valued SSB modulation ("electrical SSB") is equivalent to real valued DSB modulation with additional sideband suppression by optical filtering. Apart from hardware complexity and aspects like imperfections of the hardware components, whose analysis is beyond the scope of this paper, the only significant difference between these two approaches is the fact, that electrical SSB modulation introduces an additional phase rotation by $-\arg \bar{x}_{c}$, which has to be corrected.

Fig. 5 shows the simulated averaged estimated channel of an optical OFDM system using electrical SSB modulation, with an FFT length of 2048 and 511 used subcarriers with a bitrate of $10.7 \mathrm{~Gb} / \mathrm{s}$ using Quaternary Phase Shift Keying (QPSK) over $400 \mathrm{~km}$ of Standard Single Mode Fibre at a wavelength of $1550 \mathrm{~nm}$ and a cyclic prefix with length $1 / 4$ of the core symbol duration. Ideal linear modulation with $\bar{x}_{\mathrm{c}}=0.5+0.5 j$ and square-law detection was assumed. The physical channel $H(j \omega)$ is plotted in dashed line style. The ripple visible is caused by the linear phase lowpass filtering performed in the simulation, the phase response has been adjusted for the linear part for 


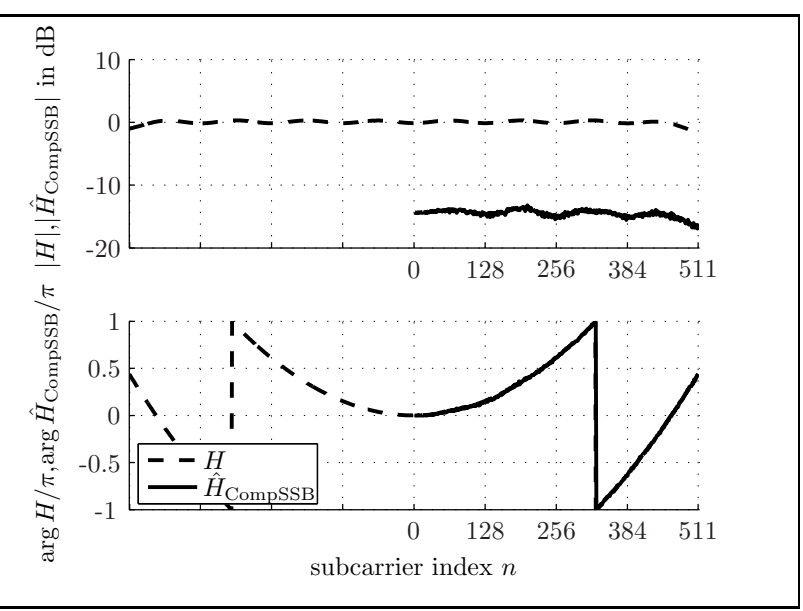

Fig. 6. Channel estimation in an optical OFDM system using compatible SSB modulation.

better presentability. The estimation $\hat{H}_{\mathrm{SSB}}(j \omega)$ has been averaged over 32 random QPSK training symbols. It can be seen that the magnitude $\left|\hat{H}_{\mathrm{SSB}}\right|$ is approximately $3 \mathrm{~dB}$ smaller that the magnitude of the physical channel, which can be explained by $\left|\tilde{x}_{\mathrm{c}}\right|=1 / \sqrt{2}$, the deviations over the subcarrier index, i.e., over frequency are caused by the interference terms mentioned earlier, resulting from the squared magnitude detection. The phase response shows an offset $-\pi / 4$, which is identical to $-\arg \bar{x}_{\mathrm{c}}$. Apart from that, the quadratic phase response of the physical channel is reproduced clearly.

\subsection{Application to compatible SSB}

The above result (55) applies to any single sideband signal. But for the compatible single sideband modulation (45), $\tilde{\varsigma}^{\prime}(t)$ is not the signal of interest, but $s(t)=\left|\varsigma_{\mathrm{c}}(t)\right|$. Due to this, the derivation of a closed form solution of the equivalent baseband channel for an arbitrary channel $h_{\mathrm{c}}(t)$ is not possible to our knowledge. Fig. 6 shows the simulated averaged estimated channel of an optical OFDM system with identical parameters as above, but using compatible SSB modulation, magnitude detection and $\bar{x}_{\mathrm{c}}=0.5$. It can be seen that the general character of the fibre optical channel is maintained in this scenario: The magnitude of the frequency response exhibits an offset, but no significant deviations. The phase response follows the physical channel and shows only a minor systematic deviation, which is depicted in Fig. 7. This systematic deviation is small enough to justify the use of a channel with quadratic phase response as equivalent baseband channel for compatible SSB modulation, e.g. as reference for perfect channel knowledge in comparison of OFDM channel estimation algorithms.

\section{Conclusions}

In this paper, the derivation of an equivalent baseband channel for systems using envelope detection was shown.

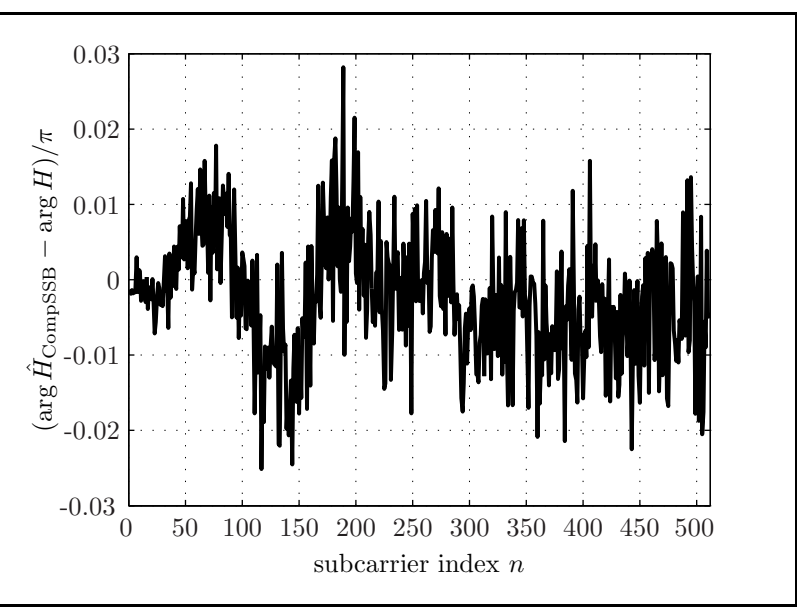

Fig. 7. Deviation of channel estimation in an optical OFDM system using compatible SSB modulation from the physical channel.

First, the system model was presented, then expressions for the output of square-law and magnitude detectors were presented. The retrieved equivalent channel descriptions were then evaluated for different physical channels. Finally, complex-valued modulation, in particular single sideband and compatible single sideband modulation was introduced and the channel model was extended accordingly. It was shown that linear modulation schemes can be represented satisfactorily with the considerations made here, while this is not possible for nonlinear schemes such as compatible single sideband modulation.

\section{Acknowledgement}

This work was supported by the German Research Foundation (DFG) under grant Ka814/19.

\section{References}

[1] Shieh, W.; Athaudage, C.: Coherent Optical Orthogonal Frequency Division Multiplexing. IET Electronics Letters $\mathbf{4 2}$ (2006), 587-588.

[2] Djordjevic, I. B.; Vasic, B.: 100-Gb/s transmission using Orthogonal Frequency-Division Multiplexing. IEEE Photonics Technology Letters 18 (2006), 1576-1578.

[3] Lowery, A.; Armstrong, J.: Orthogonal Frequency Division Multiplexing for Optical Dispersion Compensation. IEEE Optical Fiber Communication Conference and Exposition (OFC) 2007, Anaheim, USA, March 2007.

[4] Leibrich, J.; Ali, A.; Rosenkranz, W.: Optical OFDM as a Promising Technique for Bandwidth-Efficient High-Speed Data Transmission over Optical Fiber. 12th International OFDM Workshop (InOWo) 2007, Hamburg, Germany, August 2007.

[5] Schuster, M.; Randel, S.; Bunge, C.; Lee, S.; Breyer, F.; Spinnler, B.; Petermann, K.: Spectrally Efficient Compatible Single-Sideband Modulation for OFDM Transmission With Direct Detection. IEEE Photonics Technology Letters 20 (May 2008), 670-672.

[6] Rohde, U.; Whitaker, J.: Communications Receivers: DSP, Software Radios and Design. Columbus, OH, USA: The McGraw-Hill Companies, 2000. 
[7] Bennett, W. R.: Envelope Detection of a Unit-Index Amplitude-Modulated Carrier Accompanied by Noise. IEEE Transactions on Information Theory IT-20 (1974), 723-728.

[8] Kammeyer, K.-D.: Nachrichtenübertragung. 4. ed. Wiesbaden, Germany: Vieweg+Teubner Verlag, Reihe Informationstechnik, March 2008.

[9] Agrawal, G. P.: Fiber-Optic Communication Systems. 3. ed. New York, USA: John Wiley \& Sons, Inc., 2002.

[10] Wang, J.; Petermann, K.: Small Signal Analysis for Dispersive Optical Fiber Communication Systems. IEEE Journal of Lightwave Technology 10 (Jan 1992), 96-100.

[11] Yonenaga, K.; Takachio, N.: Dispersion Compensation for homodyne detection systems using a $10 \mathrm{~Gb} / \mathrm{s}$ optical PSK-VSB signal. IEEE Photonics Technology Letters 7 (August 1995), 929-931.

[12] Powers, K. H.: The Compatibility Problem in Single-Sideband Transmission. Proceedings of the IRE 48 (August 1960), 1431-1435.

[13] Kettel, E.: Einseitenbandsignale und das Problem einer Einseitenbandmodulation, bei der die Nachricht in der Enveloppe liegt. Telefunken-Zeitung (1964), 247-259.

[14] Voelcker, H.: Demodulation of Single-Sideband Signals Via Envelope Detection. IEEE Transactions on Communication Technology 14 (1966), 22-30.

Henning Paul received the masters degree (Dipl.-Ing.) in electrical engineering in 2007 from the University of Bremen, Germany. He is now a research assistant in the Department of Communications Engineering (ANT) at the Institute of Telecommunications and High-Frequency Techniques at the University of Bremen and is currently working towards his Ph.D. degree in the field of Orthogonal Frequency Division Multiplexing for fibre optical channels.

Karl-Dirk Kammeyer received the masters degree (Dipl.-Ing.) in electrical engineering from Berlin University of Technology, Berlin, Germany, in 1972, and the Ph.D. degree (Dr.-Ing.) from Erlangen University, Erlangen, Germany, in 1977. From 1972 to 1979, he worked in the field of data transmission, digital signal processing, and digital filters at the Universities of Berlin, Saarbrücken, and Erlangen, all in Germany. From 1979 to 1984 , he was with Paderborn University, Paderborn, Germany, where he was engaged in the development of digital broadcasting systems. During the following decade, he was Professor for Digital Signal Processing in Communications at Hamburg University of Technology, Hamburg, Germany. In 1995, he was appointed Professor for Telecommunications at the University of Bremen, Bremen, Germany. His research interests are digital (adaptive) systems and signal processing in mobile communication systems (multicarrier and MIMO systems). He holds 14 patent families, and has published two course books as well as over 200 technical papers. 